\title{
Phlegmasia cerulea dolens as a complication of percutaneous insertion of a vena caval filter
}

\author{
LARRY JAY FEINMAN, DO \\ ALAN JAY MELTZER, MD
}

Unsuccessfully treated

iliofemoral venous thrombosis can result in pulmonary embolism, phlegmasia cerulea dolens, and post-thrombotic syndrome. Phlegmasia cerulea dolens is characterized by tense swelling of the lower extremity with tenderness of the thigh over the femoral vein, mottling of the limb, and absent distal pulses. Without treatment, phlegmasia cerulea dolens can progress to cause venous gangrene and ultimate limb loss. In the reported case, phlegmasia cerulea dolens developed in a middle-aged woman with metastatic thyroid carcinoma following Greenfield filter placement via the femoral vein after heparinization for deep-vein thrombophlebitis had failed.

When deep-vein thrombophlebitis affects the iliofemoral system, it can produce a wide range of clinical findings, from asymptomatic, free-floating thrombus ${ }^{1}$ to venous gangrene. ${ }^{2}$

Phlegmasia alba dolens, or milk leg, occurs when the thrombus results in unilateral pain, swelling, tingling, and weakness of the extremity and tenderness over the superficial veins. As the disease progresses and edema interferes with arterial inflow, phlegmasia cerulea dolens occurs and venous gangrene and ultimately limb loss can result. ${ }^{2}$ Ten percent of patients with iliofemoral thrombosis develop phlegmasia cerulea dolens. ${ }^{2}$ Risk factors for iliofemoral venous thrombosis include previous venous thrombosis, ${ }^{1}$ oral contraceptive use ${ }^{3}$ recent operation or trauma, medical illness, and malignancy. ${ }^{4}$

Treatment is warranted to prevent pulmonary embolism and development of postphlebitic syndrome and to eliminate the risk of limb loss in phleg- masia cerulea dolens. ${ }^{5}$ The mainstay of treatment is bedrest, leg elevation, compresses, and anticoagulation. ${ }^{1}$ However, in patients who fail to respond to anticoagulation therapy or in whom anticoagulation is contraindicated, or in patients with impending limb loss, iliofemoral venous thrombectomy is indicated.

Untreated iliofemoral thrombosis results in an $18 \%$ mortality rate, pulmonary embolism incidence of $60 \%$ to $70 \%$, and post-thrombotic syndrome rate of $90 \%$. With heparin therapy, these statistics improve to mortality, pulmonary embolism, and postthrombotic syndrome rates of $1 \%, 12 \%$, and $50 \%$, respectively. ${ }^{6}$

In the case presented here, we describe a woman who progressed from deep venous thrombosis to phlegmasia cerulea dolens. The treatment consisted of conservative management and when that failed, surgical therapy. Despite all efforts, the patient succumbed to complications of pulmonary embolism.

\section{Report of case}

A 47-year-old woman, who several weeks earlier had been discharged with a diagnosis of thyroid carcinoma that had metastasized to the lymphatic nodes and pericardium, was readmitted because of dyspnea. Since her discharge, she had been doing well, until she had noticed right calf tenderness about six days prior to this admission, and then the sudden appearance of a nonproductive cough, fever, palpitations, and sweating four days later.

Beside the metastatic thyroid carcinoma, the previous medical history was significant for hypertension. The patient was taking Cytomel, Tenoretic, and potassium replacement medications. She did not abuse alcohol, and she had a 30 pack-year smoking history. 


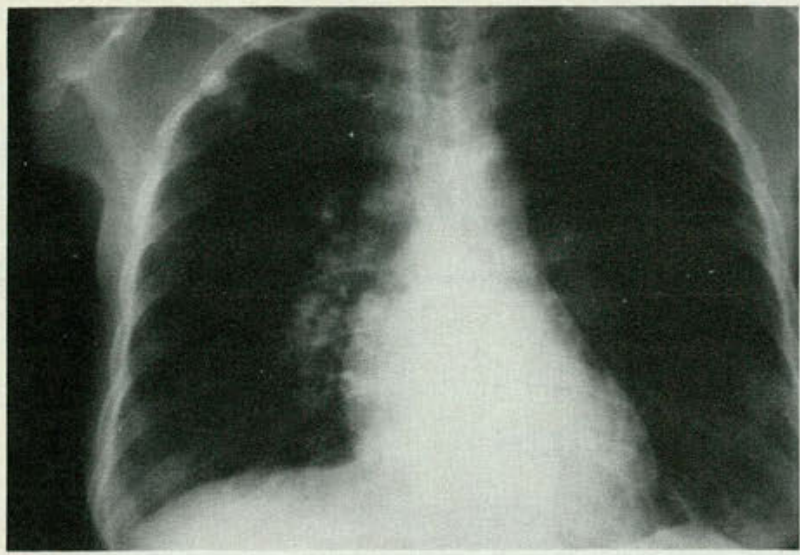

Fig 1. Admission chest film demonstrates basilar pleural reactions bilaterally and acute interstitial changes.

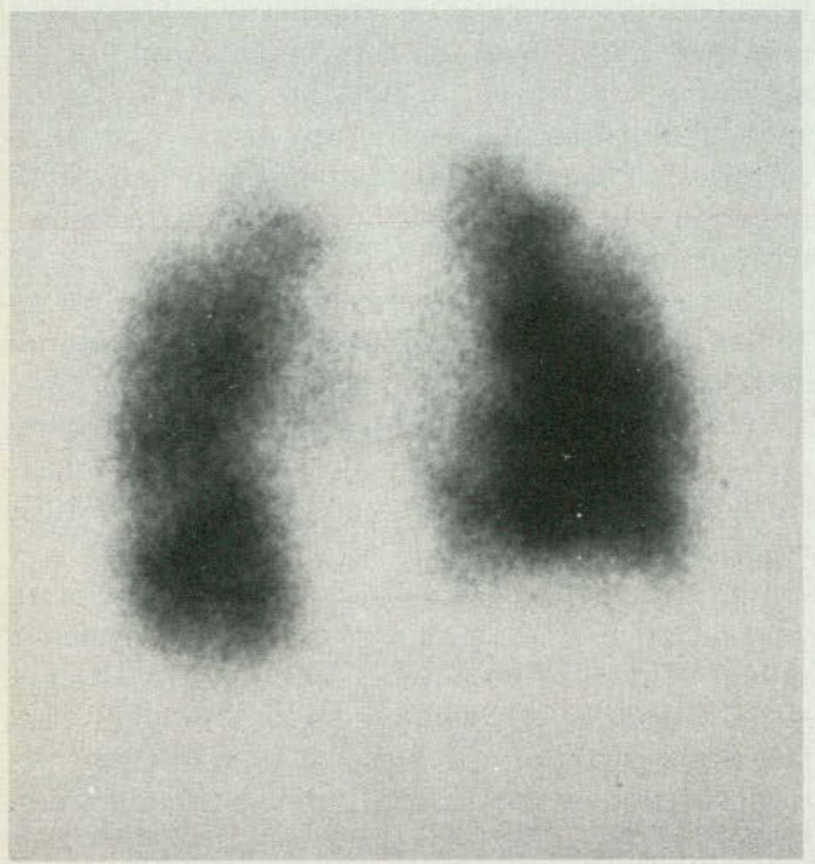

Fig 2. Perfusion lung scan demonstrates multiple defects.

On physical examination, the patient was anxious and in moderate respiratory distress. Her temperature was $100.2^{\circ} \mathrm{F}$. She had a well-healed scar from recent thyroid surgery. The heart rate was regular at 100 beats per minute, and the blood pressure was $100 / 60 \mathrm{~mm} \mathrm{Hg}$. Auscultation of the lungs revealed diffuse rhonchi and decreased breath sounds in the right base. The abdomen was normal. Extremity examination disclosed a tender right calf, with a positive Homans' sign. The right leg was warm to the touch. The left leg was normal.

The admission chest roentgenogram showed acute interstitial changes and bilateral basilar pleu-

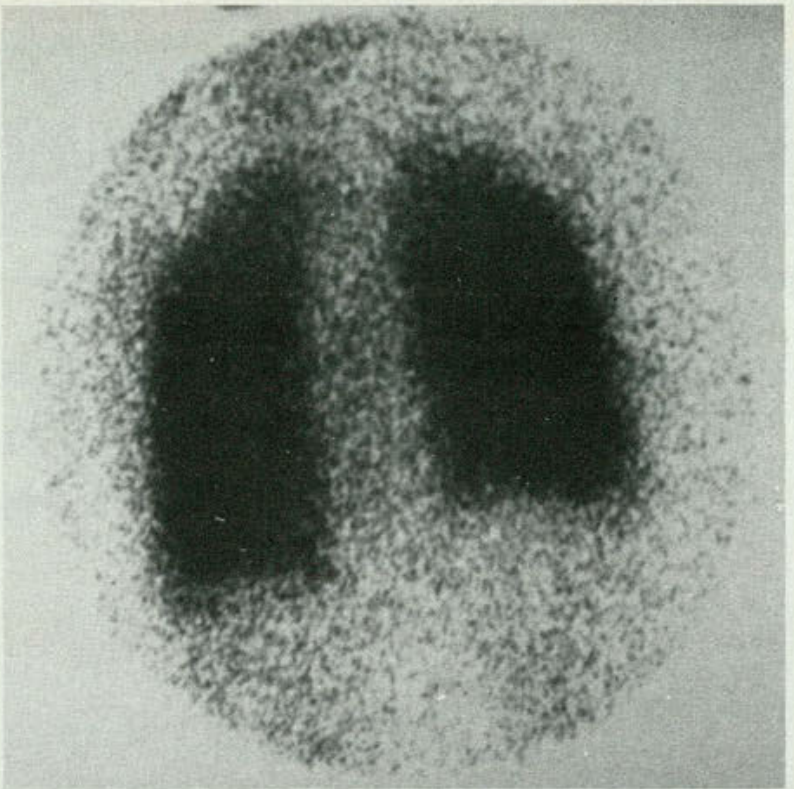

Fig 3. Defects are mismatched on ventilation lung scan.

ral reactions (Fig 1). Initial electrocardiography demonstrated poor $\mathrm{R}$-wave progression across the precordium and T-wave inversion precordially. The WBC was $11,900 / \mathrm{cu} \mathrm{mm}$, without a differential shift. The hemoglobin and hematocrit values were $10.6 \mathrm{~g} / \mathrm{dL}$ and $32.1 \%$, respectively. The platelet count and baseline chemistry values were normal. Admission arterial blood gas determinations revealed respiratory alkalosis, as follows: $\mathrm{pH}, 7.52$; $\mathrm{pO}_{2}, 70 \mathrm{~mm} \mathrm{Hg} ; \mathrm{pCO}_{2}, 31 \mathrm{~mm} \mathrm{Hg}$; and $\mathrm{HCO}_{3}, 25$ $\mathrm{mm} \mathrm{Hg}$.

With a tentative diagnosis of pulmonary embolism on the day of admission, the patient underwent perfusion and ventilation lung scanning, which demonstrated multiple unmatched perfusion defects (Figs 2 and 3). Next, emergency pulmonary angiography showed multiple, bilateral emboli (Figs 4 and 5).

Right-sided lower extremity venography performed concurrently with the pulmonary angiography demonstrated calf vein thrombosis with extension into the distal superficial femoral vein (Figs 6 and 7). Neither the proximal venous circulation or the vena caval system was involved with the thrombus (Figs 8 and 9).

The patient stabilized on initial heparin therapy. However, three days after treatment was started, she began to experience worsening pulmonary function, and recurrent pulmonary embolism was diagnosed. Using the Seldinger technique and a right femoral approach, a Greenfield vena caval filter was placed by the invasive radiologist into the infrarenal vena cava. 


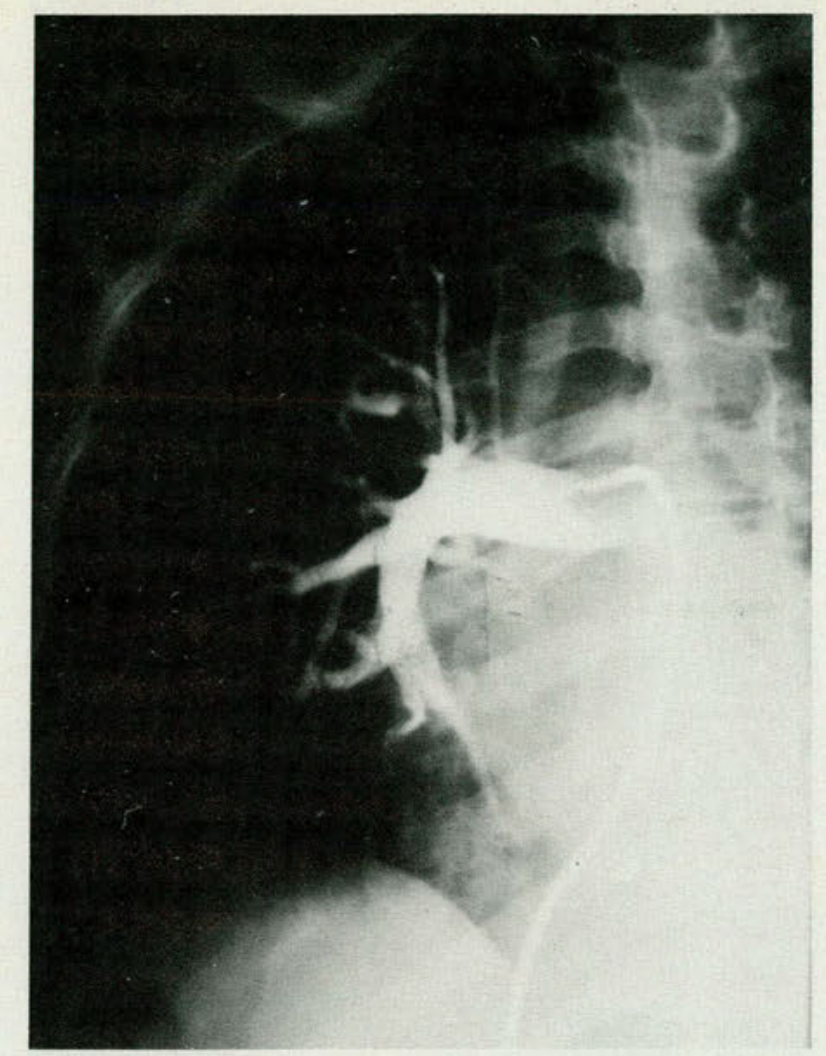

Figs 4. and 5. Pulmonary angiograms show multiple emboli(Fig 4, right-sided anteroposterior projection; Fig 5 , left-sided oblique projection).

Two hours after filter placement, the patient began to complain of severe pain in her right leg. Her blood pressure fell to $80 / 60 \mathrm{~mm} \mathrm{Hg}$, and she became tachycardic. Her leg was noted to be red and edematous, with intact pulses. The heparin had been discontinued after placing the filter; it was then restarted. Eighteen hours after placement, the leg became progressively more edematous, the pulses were lost, and the leg became cold.

The diagnosis of phlegmasia cerulea dolens was made, and the patient underwent right lower extremity arteriography to rule out acute arterial occlusion. The arterial vasculature was open, but the flow was quite slow. This was thought to be caused by the significant edema in the limb.

Twenty-four hours after placement of the filter, the patient was taken to surgery. The right femoral vein was noted to be filled with clot, and the vein was freed from the surrounding tissue and incised. A massive amount of thrombus was removed from the proximal venous system with Fogarty thrombectomy catheters and suction. Good backbleeding was noted. The lower extremity was

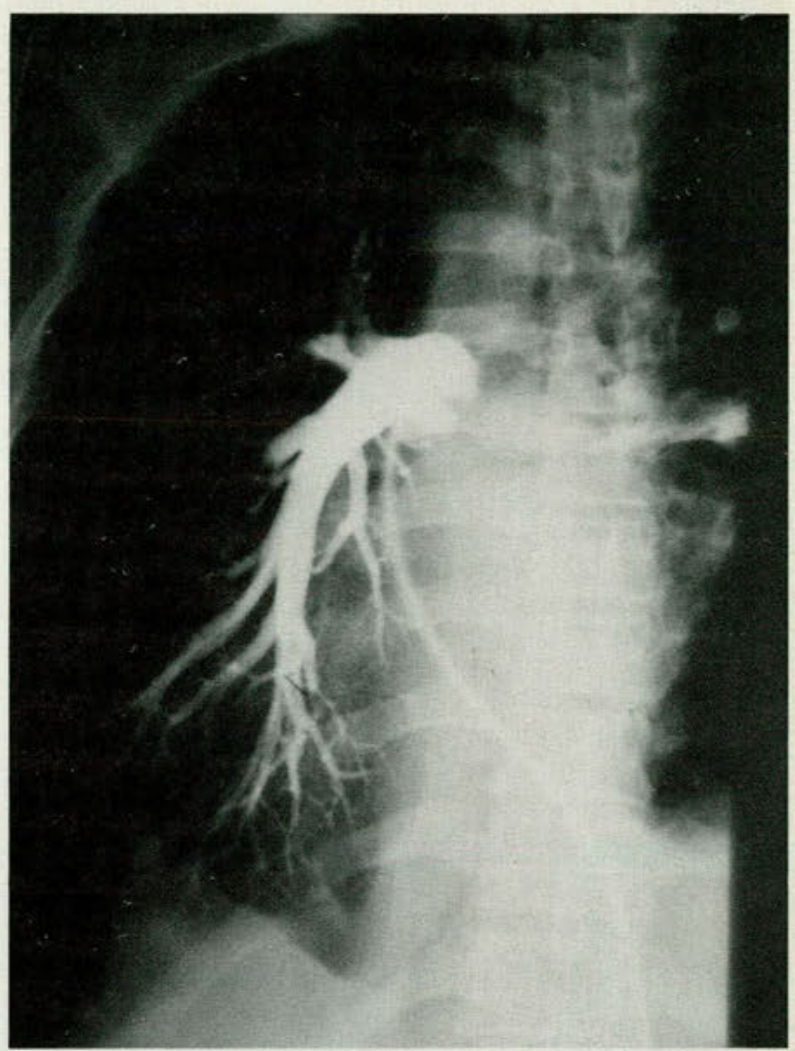

milked to express thrombus from the distal veins, and, after this was done, there was brisk venous bleeding. The vein was closed, and before completion of the procedure, an extensive lower leg fasciotomy was performed to release all compartments of the leg.

Postoperatively, the patient's condition progressively worsened and she died two days later of progressive adult respiratory distress syndrome and sepsis.

\section{Discussion}

Iliofemoral venous thrombosis differs from lower extremity deep-vein thrombosis in a few respects. Seventy-five percent of cases of iliofemoral venous thrombosis occur in the left leg, because of the anatomy of the left iliac vein. In the pelvis, the vein crosses under the right iliac artery, at which point it is subject to compression. In contrast to deepvein thrombosis, which generally recanalizes, iliofemoral venous thrombosis has a higher rate of failure to recanalize. ${ }^{7}$ Veins that are completely occluded, such as those in iliofemoral thrombosis, rarely recanalize, and never before five months postthrombosis. $^{8}$

One goal of successful therapy is to prevent the postphlebitic syndrome, which is manifested by edema, venous stasis ulcers, and venous claudica- 


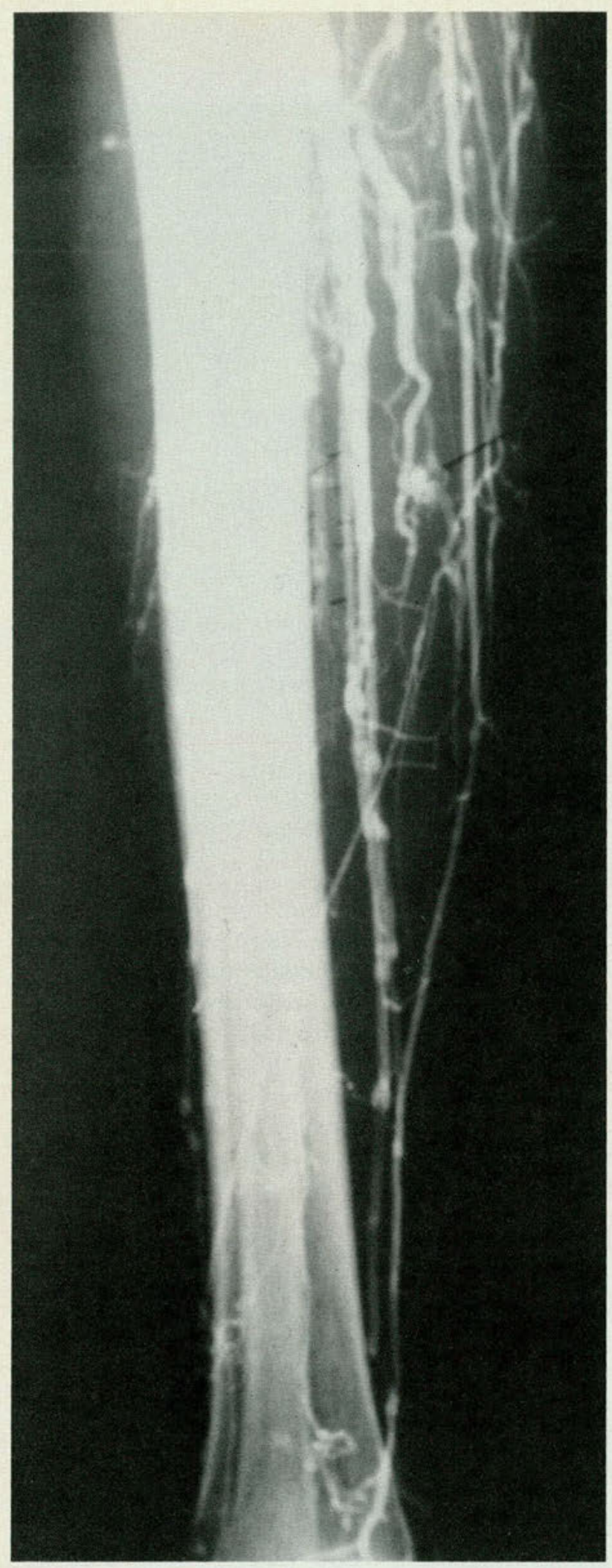

Fig 6. Venogram of right lower leg reveals extensive calf vein thrombosis.

tion. ${ }^{9}$ The goal in phlegmasia cerulea dolens is to eliminate the risk of limb loss. ${ }^{5}$

The experience with thrombolytic therapy has

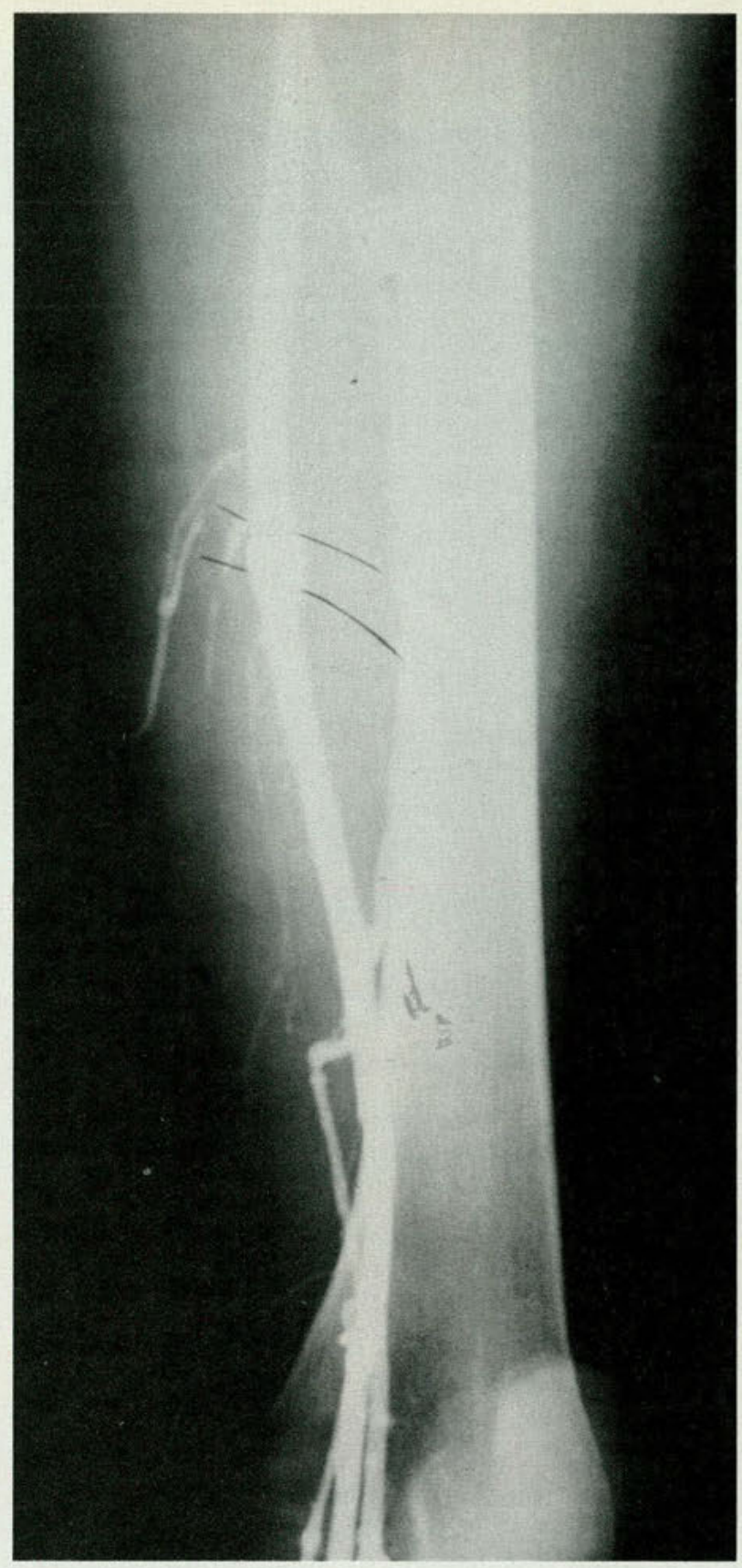

Fig 7. Upper right leg venogram demonstrates thrombus in the superficial femoral vein.

been limited, and it has not proved to be superior to the standard treatment. In addition, there has been a high incidence of side effects and bleeding complications with this mode of therapy when applied to iliofemoral thrombosis. ${ }^{4,10}$ The modality of choice for prevention of pulmonary embolism is heparinization. If this procedure fails, or if the patient cannot tolerate anticoagulation, placement of a Greenfield filter is the next preferred treatment. ${ }^{6}$ The only time thrombectomy is justified to prevent pulmonary embolism is when a free-floating throm- 


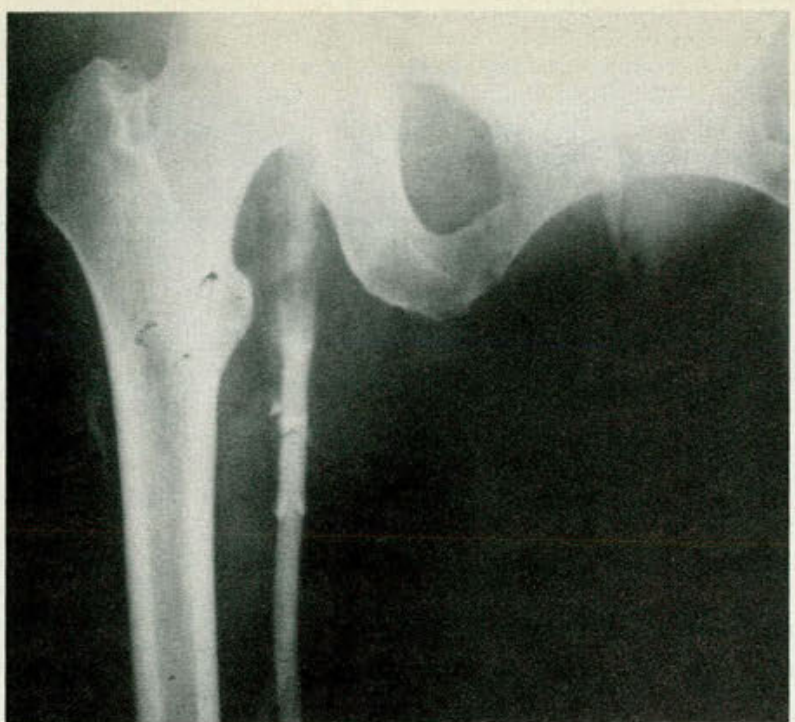

Fig 8. Venogram of proximal venous circulation demonstrates that the common femoral vein is free of thrombus.

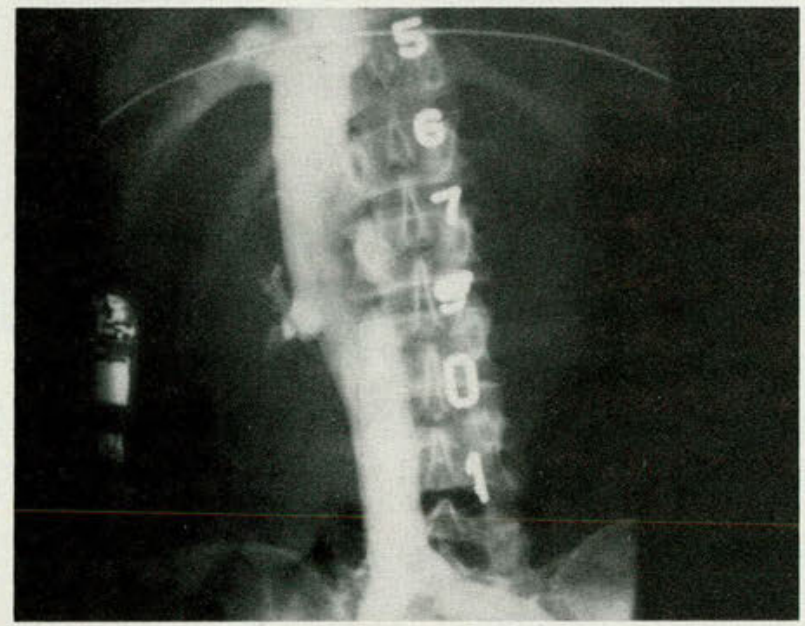

Fig 9. Venocavogram demonstrates patent caval system.

bus is demonstrated in the iliofemoral system. ${ }^{9}$

The duration of symptoms prior to operative intervention is important. Most investigators are in agreement that thrombectomy is impossible after 14 days of symptoms. ${ }^{4,10}$ In one study, ${ }^{9}$ good or fair results were obtained in all 10 patients operated on within 48 hours of onset of symptoms, while $91 \%$ of patients who were operated on within seven days of symptom onset had excellent results in another study. ${ }^{4}$

The surgical technique involves complete removal of thrombus from the entire venous system. The femoral vein is dissected free, and the superficial femoral, deep femoral, and saphenous branches are mobilized. The proximal thrombus is removed using Fogarty venous thrombectomy catheters, and the distal thrombus is removed by manual com- pression of the $\operatorname{leg}^{2}$ or by wrapping an elastic band around the limb. ${ }^{5}$ Completion venography should be performed to document complete clearance of the thrombus. ${ }^{11}$ In one study ${ }^{4}$ of 12 patients with incomplete clearance during operative venography, 10 had partial or total occlusion at phlebography performed seven to 10 days postoperatively.

Our patient had developed deep-vein thrombophlebitis six days prior to admission, but on admission venography, she demonstrated only calf vein thrombosis. The most proximal extension of the deep-vein thrombophlebitis was to the distal superficial femoral vein. The iliofemoral system was spared. She developed phlegmasia cerulea dolens as a complication of Greenfield filter placement via the femoral vein.

Her risk factors for development of iliofemoral thrombosis were malignancy and recent surgery. In addition, she underwent venography three days prior to development of phlegmasia cerulea dolens, which may be an additional risk factor. ${ }^{12}$ Table 1 outlines the most common risk factors for iliofemral venous thrombosis.

Phlegmasia cerulea dolens is characterized by tense swelling in the leg, mottling of the limb, coolness to the touch, and absence of distal pulses. If not treated expeditiously, it can lead to venous gangrene and limb loss.

The etiology of arterial insufficiency is thought to be extrinsic pressure secondary to the tense swelling of the limb from the venous occlusion. ${ }^{13}$ This was shown quite well in a study ${ }^{14}$ in which intramuscular pressure catheters were inserted into the anterior and posterior tibial compartments of a patient with phlegmasia cerulea dolens. The initial

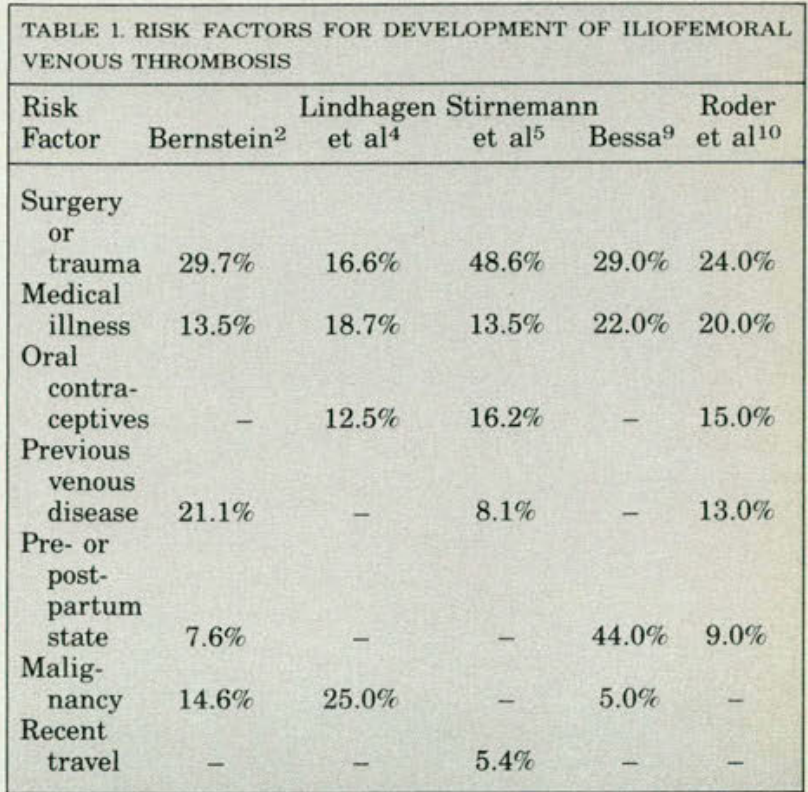


pressures were 49 and $57 \mathrm{~mm} \mathrm{Hg}$ in the anterior and posterior tibial compartments, respectively. Immediately after surgery, the pressures were 30 and $36 \mathrm{~mm} \mathrm{Hg}$, respectively, and 16 hours postoperatively, the pressures were 16 and $20 \mathrm{~mm} \mathrm{Hg}$, respectively.

\section{Summary}

Phlegmasia cerulea dolens results in $10 \%$ of patients with iliofemoral thrombosis. The massive edema caused by venous occlusion results in compromise of the arterial flow and limb loss can occur. In patients whose limbs are not lost, postphlebitic syndrome and venous gangrene can result. Aggressive treatment involves anticoagulation, bed rest, and elevation. In patients who fail this therapy, venous thrombectomy is warranted.

We presented a case of a woman with metastatic thyroid carcinoma who developed deep venous thrombosis which progressed to phlegmasia cerulea dolens. Despite aggressive surgical management, the patient succumbed to the respiratory complications of a pulmonary embolus.

1. Stephens GL: Current opinion on iliofemoral venous thrombectomy. Am Surg 1976;42:108-115.

2. Bernstein EF: Operative management of acute venous thrombosis, in Rutherford RB (ed):Vascular Surgery. WB Saunders Co, 1984, pp 13671384.
3. Coddington CC, Drake TS: Phlegmasia cerulea dolens as a complication of short-course oral contraceptives for dysfunctional bleeding. South Med J 1982;75:377-378.

4. Lindhagen J, Haglund M, Haglund U, et al: Iliofemoral venous thrombectomy. J Cardiovasc Surg 1978;19:319-327.

5. Stirnemann P, Althaus U, Kirchhof B, et al: Early phlebographic results after iliofemoral venous thrombectomy. Thorac Cardiovasc Surg 1984;32:299-303.

6. Greenfield LJ, Alexander EL: Current status of surgical therapy for deep vein thrombosis. Am J Surg 1985;150:64-70.

7. Rutherford RB: Role of surgery in iliofemoral venous thrombosis. Chest 1986;89(suppl 5):434S-437S

8. Warren R: Behavior of venous thrombi: Historical observations. Arch Surg 1980;115:1151-1154.

9. Bessa JC: Femoral and iliofemoral thrombectomy to prevent chronic venous insufficiency: Follow-up of 18 patients. J Cardiovasc Surg $1986 ; 27: 443-446$.

10. Roder OC, Lorentzen JE, Hansen HJB: Venous thrombectomy for iliofemoral thrombosis: Early and long-term results in 46 consecutive cases. Acta Chir Scand 1984;150:31-34.

11. Dale WA, Allen TR: Unusual problems of venous thrombosis. Surgery $1985 ; 78: 707-722$.

12. Laerum F, Laake K: Phlegmasia cerulea dolens after pelvic phlebography with meglumine metrizoate. Europ J Radiol 1981;1:117-118. 13. Lipchik EO, Altman DP: Phlegmasia cerulea dolens. Radiology 1979;133:81-82.

14. Saffle JR, Maxwell JG, Warden GD, et al: Measurement of intramuscular pressure in the management of massive venous occlusion. Sur gery 1981;89:394-397.

From the Department of Vascular Surgery, Metropolitan Hospital, Parkview Division, Philadelphia.

Reprint requests to Dr Feinman, Suite A-01, 1331 E Wyoming Avenue, Philadelphia 19124. 